\title{
Optimisation of Preventive Maintenance Scheduling under Heterogeneous Policy
}

\author{
Shawulu Hunira Nggada \\ Department of Computer Science, \\ Faculty of Computing and Informatics, \\ Namibia University of Science and Technology, \\ Windhoek, Namibia. \\ snggada@nust.na
}

\begin{abstract}
The effect of maintenance activities on the component of a system could either be perfect or imperfect. A perfect maintenance implies that the condition of the component is as good as new while imperfect implies that its condition has improved to a certain degree. Real engineering system problems in this domain usually consist of components with varying maintenance policies; perfect and imperfect. It is usually easier to optimise or analyse a system when subjected to homogeneous preventive maintenance (PM) policy; either solely perfect or imperfect. This paper investigates the optimisation of a heterogeneous (both perfect and imperfect) PM policy by using respective evaluation models and establishing a variant of the non-dominated sorting genetic algorithm (NSGA) II for the problem.
\end{abstract}

Keywords: Preventive maintenance, CoMI, optimisation, genetic algorithm, unavailability.

\section{Introduction}

Maintenance is known to improve the reliability and availability of engineering systems. According to Zhao et al [1], system reliability can be improved significantly if defects are detected during planned inspections. Nggada et al [2] also showed that maintenance actions improve the reliability and availability of a system. Typically, maintenance actions are performed on the components of the system and the overall system reliability or availability is the combination of function (reliability or availability) evaluations at its constituent components level.

Under preventive maintenance, maintenance is planned and usually performed periodically. The time interval at which a component is scheduled for maintenance is referred to as preventive maintenance time or simply PM time $\mathrm{T}_{\mathrm{p}}$, and the time at which maintenance is performed is referred to as PM stage. The preventive maintenance time of a given component is a function of the system's shortest PM interval T. The relationship is represented as shown in equation 1 [3].

$T_{p i}=\alpha_{i} T$

Where: $T_{p i}$ is the PM time for component $i$

$\alpha_{i}$ is referred to as the coefficient of maintenance interval (CoMI)

$T$ is the system's shortest PM interval

Each component of the system can have numerous potential PM times and this is sequel to variations of its CoMIs[2]. CoMI is an integer variable with minimum and 
maximum value as discussed in section 3 of this paper. Hence, if the potential PM time of the $i$-th component is represented by $p o T_{p i}$, then the following holds.

$p o T_{p i}=\left\{T_{p i 1}, T_{p i 2}, T_{p i 3}, T_{p i 4}, T_{p i 5}, \ldots, T_{p i(n-1)}, T_{p i k}\right\}$

Where: $\quad k$ is the total number of potential PM times for the $i$-th component

Equation 2 simply denotes the various options of PM time for a single component (the $i$-th component) of the system. The PM time of the component will be one of those indicated in equation 2 and therefore, the following holds.

$$
\mathrm{A} t_{p} \varepsilon p o T_{p i}, \mathrm{E}_{1} t_{p} \varnothing T_{p i}=t_{p}
$$

The potential PM schedule (PMS) of a systems is usually represented as shown in equation 4.

$P M S=\left\{T_{p 1}, T_{p 2}, T_{p 3}, T_{p 4}, T_{p 5}, \ldots, T_{p(m-1)}, T_{p m}\right\}$

Where: $m$ is the total number of components under the PM policy

From equation 2, it follows that each element of the PMS in equation 4 is a selection from its potential PM times. For instance, considering a simple and trivial case of a system with 3 components and each having 3 potential PM times, this would mean that there are $3^{3}(27)$ potential maintenance schedules[15]. For a larger system or even the system with three components but having numerous potential PM times, it is practically infeasible to manually enumerate the PM schedules. Additionally, the evaluations of the PM schedules that would guide the engineer in selecting the schedule or schedules that meet design requirements are also difficult to manually perform. This becomes an automated optimisation problem and this is what this paper addresses by considering a heterogeneous PM policy using unavailability and cost as objective functions.

It is usually easier to optimise homogeneous PM policy where the effect of maintenance actions on each component of the system is assumed to follow same pattern. For instance the effect could be assumed to be perfect as considered by Nggada et al [2] or imperfect. In this paper the effect of maintenance actions on certain components is assumed to be perfectwhile the assumption on the rest is imperfect. Thus, the reminder of this paper is structured as follows. Section 2 establishes the evaluation models for heterogeneous PM policy. In section 3 the optimisation problem is defined, and section 4 presents the case study on which the evaluations in section 5 are performed. Finally section 6 draws conclusions.

\section{Evaluation Models}

The evaluation models for the heterogeneous PM policy are established with respect to the proportional age reduction (PAR) model. The PAR model assumes that each maintenance activity reduces proportionally the age gained from previous maintenance [4]. This implies that each PM activity is assumed to only reduce a portion of the component age. The PAR is dependent on the effectiveness of the maintenance activity referred to as improvement factor $f$, where $f$ lies between 0 and 1 inclusive $(0 \leq f \leq 1)$. When $f=1$ the maintenance is a perfect one [5]. A maintenance activity conducted at the $i$-th time $t_{i}$, with an improvement factor $f_{i}$ for a given component of a system reduces the component age $W_{i}$ as shown in equation $5[4,6]$.

$$
W_{i}^{+}=\left(1-f_{i}\right) t_{i}
$$


Where $t_{i}$ is the time at which the $i$-th maintenance is carried out. $W_{i}^{+}$is known as the effective age of the component. The plus sign symbolises that the effect of age reduction applies only after the PM activity.To establish the evaluation models, a second assumption in this paper is that component failures follow the Weibull distribution.

\subsection{Perfect Preventive Maintenance (PPM) Models}

Under prefect preventive maintenance policy there is no repair and this simplifies the evaluation models. The availability and cost models are discussed next.

2.1.1. PPM Availability and Unavailability Model: The assumption under PPM is that there is no repair and therefore, availability is synonymous to reliability. Hence the availability model is as shown in equation 6 [2] and unavailability in equation 7.

$$
A_{p p m}(t)=\exp \left[-n\left(\frac{T_{p}}{\Theta}\right)^{\beta}\right] \exp \left[-\left(\frac{t-n T_{p}}{\Theta}\right)^{\beta}\right] \quad ; n T_{p} \leq t \leq(n+1) T_{p}
$$

Where: $A_{p p m}$ is availability under PPM

$t$ is the time under consideration

$T_{p}$ is PM time of the component under consideration

$n$ is the total number of PM times for the component

$\beta$ is Weibull shape parameter

Өis the Weibull scale parameter

The work in this paper also assumes that component was new at the start of the maintenance policy. Therefore, $\gamma$ (location parameter) assumes value 0 and this is exactly why it does not appear in equation 6 .

$U_{p p m}(t)=1-A_{p p m}(t)(7)$

Where: $U_{p p m}$ is unavailability under PPM

2.1.2. PPM Cost Model: The total preventive maintenance cost depends on the total number of PM stages for a given component. With the assumption that no repair is carried out under PPM policy, the component total cost model becomes a simple one. Considering the $i$-thcomponent of a system, its total cost is as shown in equation 8 . This is a basic cost model and could be modified or extended depending on the problem domain.

$C_{p c i}=n_{i} C_{p p m i}+C_{c i}$

Where: $C_{p c i}$ is the total cost for the $i$-th component under PPM

$C_{p p m i}$ is the cost of performing PPM for the $i$-th component

$C_{c i}$ is the unit cost of the $i$-th component

$n_{i}$ is the total number of PM stages for the $i$-th component

Using equation 8 , the total system $\operatorname{cost} C_{P P M s}$ under PPM is established as:

$C_{P P M s}=\sum_{i}^{m} C_{p c i}$ 
Where $m$ as earlier mentioned is the number of system components identified for PPM.

\subsection{Imperfect Preventive Maintenance (IPM) Models}

Under IPM policy, repair of components is taken into account. However, one of objectives of IPM is to improve availability either through quick but effective repair or reducing to a very minimal level the occurrence of failure that will infer corrective maintenance. Hence, the third assumption in this paper is that no failure that will bring the system to halt resulting into corrective maintenance occurs in-between PM times. Sequel to this, minimal repair [5] is considered.

2.2.1. IPM Availability and Unavailability Model:The availability $A_{\mathrm{ipm}}$ of a component depends on reliability and maintenance. Thus availability can be modeled using the standard availability expression shown in equation 10 [7].

$A_{\text {ipm }}=\frac{U T}{U T+D T}$

Where:UT is the up time of the component

DT is the down time of the component

Let $\mu_{\mathrm{m}}$ be the mean time for minimal repair of the component $\mu$ be the mean time to repair of the component

$\lambda(t)$ be the hazard rate (also referred to as failure rate) of the component

Then, UT and DT can be defined as:

$U T=T_{p}-\mu_{m} \int \lambda(t) d t$

$D T=\mu+\mu_{m} \int \lambda(t) d t$

Similar expression of equations 11 and 12 are found in Tsai et al [8] while Sheu et al [9] has similar expression to equation 11.

To solve for $\lambda(t)$, let the following hold;

$N(t)=\int \lambda(t) d t$

According to Birolini[10]:

$$
\lambda(t)=-\frac{d R(t) / d t}{R(t)}
$$

Using Weibull distribution, the following applies;

$d R(t)=e^{\left[-\left(\frac{t-\gamma}{\theta}\right)^{\beta}\right]} d t$ 
Since it is assumed that a component life begins at time $t=0$, the location parameter $\gamma$ takes a value 0 and therefore the above is simplified to:

$$
d R(t)=e^{-\left(\frac{t}{\theta}\right)^{\beta}} d t
$$

By using Chain Rule and hence, integrating equation 13, the following is derived.

$$
N(t)=\frac{1}{\theta^{\beta}}\left|t^{\beta}\right|
$$

The limits of the integration will be the effective age at the previous PM stage (as lower limit) and the age at the current PM stage (as upper limit). Therefore:

$$
\begin{aligned}
& N(t)=\int_{W_{j-1}^{+}}^{W_{j}} \lambda(t) d t \\
& N(t)=\frac{1}{\theta^{\beta}}\left|t^{\beta}\right|_{W_{j-1}^{+}}^{W_{j}}
\end{aligned}
$$

Where $j$ represents the $j$-th PM time. Substituting the above in both equations 11 and 12 , gives equations 16 and 17 respectively.

$$
\begin{aligned}
& U T=T_{p}-\frac{\mu_{m}}{\theta^{\beta}}\left|t^{\beta}\right|_{W_{j-1}^{+}}^{W_{j}} \\
& D T=\mu+\frac{\mu_{m}}{\theta^{\beta}}\left|t^{\beta}\right|_{W_{j-1}^{+}}^{W_{j}}
\end{aligned}
$$

Substituting equation 16 and 17 into equation 10 gives the following:

$$
A_{i p m}=\frac{T_{p}-\frac{\mu_{m}}{\theta^{\beta}}\left|t^{\beta}\right|_{W_{j-1}^{+}}^{W_{j}}}{\left(T_{p}-\frac{\mu_{m}}{\theta^{\beta}}\left|t^{\beta}\right|_{W_{j-1}^{+}}^{W_{j}}\right)+\left(\mu+\frac{\mu_{m}}{\theta^{\beta}}\left|t^{\beta}\right|_{W_{j-1}^{+}}^{W_{j}}\right)}
$$

The above equation is only a reflection of the first PM stage, and therefore for $n$ number of PM stages, it transforms into equation 18.

$$
A_{\text {ipm }}=\frac{\sum_{j=1}^{n}\left(T_{p}-\frac{\mu_{m}}{\theta^{\beta}}\left|t^{\beta}\right|_{W_{j-1}^{+}}^{W_{j}}\right)}{\sum_{j=1}^{n}\left[\left(T_{p}-\frac{\mu_{m}}{\theta^{\beta}}\left|t^{\beta}\right|_{W_{j-1}^{+}}^{W_{j}}\right)+\left(\mu+\frac{\mu_{m}}{\theta^{\beta}}\left|t^{\beta}\right|_{W_{j-1}^{+}}^{W_{j}}\right)\right]}
$$


The unavailability of a component $\mathrm{U}_{\mathrm{ipm}}$ is therefore as expressed in equation 19 .

$U_{\text {ipm }}=1-A_{\text {ipm }}$

2.2.1. IPM Cost Model:Maintenance cost models may vary and are specific to the problem domain; for instance the cost model for production industry may differ from that of an aviation industry. As mentioned earlier this paper establishes basic model from which industry specific models could evolve. The total cost of the $i$-th component of a system under IPM and taking minimal repair into account can be expressed as shown in equation 20.

$C_{c i}=C_{m r i} \sum_{j=1}^{n} N(t)+n_{i} C_{p m i}+C_{i}$

Where: $\mathrm{C}_{\mathrm{ci}}$ is the IPM total cost for the $i$-th component

$\mathrm{C}_{\mathrm{mri}}$ is the cost of minimal repair for the $i$-th component

$\mathrm{C}_{\mathrm{i}}$ is the unit cost of the $i$-th component

$\mathrm{C}_{\mathrm{pmi}}$ is the cost of performing IPM for the $i$-th component at each PM stage

$\mathrm{N}(\mathrm{t})$ in equation 13 is as derived in equation 15 and therefore, substituting it in equation 20 gives equation 21 .

$C_{c i}=C_{m r i} \sum_{j=1}^{n}\left(\frac{1}{\theta^{\beta}}\left|t^{\beta}\right|_{W_{j-1}^{+}}^{W_{j}}\right)+n_{i} C_{p m i}+C_{i}$

The total IPM cost $C_{I P M s}$ for a system is the summation of all the total IPM cost of its constituent components. This is expressed in equation 22 .

$C_{I P M S}=\sum_{i=1}^{m} \mathrm{C}_{\mathrm{ci}}$

Where: $i$ is the index of the $i$-th component of the system $m$ is the number of components in the system identified for PM

\section{Defining the Optimisation Problem}

To define the PM optimisation, constraints which guide the solution search to region of feasible solutions must first be defined. The same constraints used by Nggada et al [2] are also adopted in this paper and are described as follows.

Constraint $1(\mathrm{C} 1)$ : The shortest system PM interval $\mathrm{T}$ is chosen such that its value is less than the failure rate of the component that fails most often in the system. This is done to ensure that PM is not performed late. This is formally denoted as follows.

$\mathrm{T}<\frac{1}{\lambda_{\mathrm{H}}}$ 
Constraint 2 (C2): The PM time of each component is chosen such that it is less or equal to the average failure time (AFT) of the component. The AFT is synonymous to mean time to failure (MTTF) in the case of PPM, and to mean time between failures in the case of IPM. This is formally denoted as follows.

$\alpha_{i} T \leq \begin{cases}\frac{1}{\lambda_{i}} & ; A T F_{i} \leq R T \\ R T & ; A T F_{i}>R T\end{cases}$

Where: $i=1 . . m$, is the number of components

$\alpha_{\mathrm{i}}$ is the CoMI for the $i$-th component

The definition of the PM scheduling optimisation is thus as follows.

$\min \mathbf{F}(\boldsymbol{\alpha})=\{U(\boldsymbol{\alpha}), C(\boldsymbol{\alpha})\}$

such that:

$\alpha \in \mathbf{A}$,

$\mathrm{T}<\frac{1}{\lambda_{\mathrm{H}}}$,

$\alpha_{i} T \leq \begin{cases}\frac{1}{\lambda_{i}} & ; A T F_{i} \leq R T \\ R T & ; A T F_{i}>R T\end{cases}$

Where: A is the solution space of all CoMIs.

$\boldsymbol{\alpha}=\left\{\alpha_{1}, \alpha_{2}, \alpha_{3}, . . \alpha_{m-2}, \alpha_{m-1}, \alpha_{m}\right\}$

$U$ is the system unavailability

$C$ is the system cost

The objective functions $(U$ and $C$ ) are detailed as follows.

$U(\boldsymbol{\alpha})=O_{s}(\boldsymbol{\alpha})= \begin{cases}\left\{U_{p p m}\left(\alpha_{1}\right), U_{p p m}\left(\alpha_{2}\right), . . U_{p p m}\left(\alpha_{m}\right),\right\} & ; \text { under PPM } \\ \left\{U_{i p m}\left(\alpha_{1}\right), U_{i p m}\left(\alpha_{2}\right), . . U_{i p m}\left(\alpha_{m}\right),\right\} & ; \text { underIPM }\end{cases}$

Where: $U_{p p m}$ is the component unavailability under PPM as expressed in equation 7

$U_{i p m}$ is the component unavailability under IPM as expressed in equation 19

$O_{S}$ is the system unavailability which is evaluated using the Esary-Proschan approximation [11] as expressed in equation 23

The Esary-Proschan evaluation is applied on the minimal cut sets of the fault trees produced for the system; i.e. by HiP-HOPS analysis or any capable tool.

$O_{s}=\prod_{i=1}^{v}\left(1-\prod_{j=1}^{k}\left(1-O_{i j}\right)\right)$ 
Where: $O_{i j}$ is the evaluated unavailability function for the $i$-th component $v$ is the number of cut sets and $k$ the order of the $i$-th cut set.

$$
C(\boldsymbol{\alpha})= \begin{cases}C_{P P M s}(\boldsymbol{\alpha})=\left\{C_{p c 1}\left(\alpha_{1}\right), C_{p c 2}\left(\alpha_{2}\right), . . C_{p c m}\left(\alpha_{m}\right),\right\} & ; \text { under PPM } \\ C_{I P M S}(\boldsymbol{\alpha})=\left\{C_{c 1}\left(\alpha_{1}\right), C_{c 2}\left(\alpha_{2}\right), \ldots C_{c m}\left(\alpha_{m}\right),\right\} & ; \text { underIPM }\end{cases}
$$

Where: $C_{P P M s}$ is the system cost under PPM as expressed in equation 9

$C_{p c i}$ is the cost for the $i$-th component under PPM as expressed in equation 8

$C_{I P M S}$ is the system cost under IPM as expressed in equation 22

$C_{c i}$ is the cost for the $i$-th component under IPM as expressed in equation 21

It is also useful to note that enforcing constraint $\mathrm{C} 2$ requires further step; the maximum CoMI $\alpha_{\max }$ for any given component must be determined. The CoMI $\alpha_{i}$ for the $i$-th component should be a value such that $1 \leq \alpha_{i} \leq \alpha_{\text {imax }} \cdot \alpha_{i \max }$ for the $i$-th component is evaluated as expressed in equation 24 .

$\alpha_{\text {imax }}= \begin{cases}Q\left(\frac{A T F}{T}\right) & ; A T F_{i} \leq R T \\ Q\left(\frac{R T}{T}\right) & ; A T F_{i}>R T\end{cases}$

Where: $Q$ is the integer quotient of the division

Thus, in generating a PM individual, each constituent CoMI is a random integer between 1 and $\alpha_{\text {imax }}$ inclusive.

\subsection{Optimisation Algorithm}

To optimise the schedules of a heterogeneous PM policy, a variant of the Nondominated Sorting Genetic Algorithm (NSGA) II is developed. The developed NSGA II is similar to that defined by Nggada et al [2] for PPM optimisation and is discussed below.

\footnotetext{
i. $\quad$ Set population index $\mathrm{t}=1$.

ii. $\quad$ Set front index $\mathrm{i}=1$.

iii. $\quad$ Randomly generate an initial population $\mathrm{P}_{\mathrm{t}}$ of $\mathrm{N}$ number of $\mathrm{PM}$ individuals. This is performed by obtaining a random value of

CoMI $\quad \alpha_{i} ; \alpha_{i}=\operatorname{random}\left(1 . . \alpha_{i \max }\right)$.

iv. $\quad \forall p \in \mathrm{P}_{\mathrm{t}}$, configure the variant of the system model with $p$ by using the encoding to set the CoMI of each component and then evaluate the unavailability and cost (objective functions) of the system.

v. $\quad \forall p \in \mathrm{P}$, find $n_{p}$ number of solutions that dominate $p$, and $\mathrm{S}_{\mathrm{p}}$ set of solutions for which $p$ dominates.

vi. $\quad$ Add all $p$ with $n_{p}=0$ into the set $\mathrm{F}_{\mathrm{i}}$ (the $i$-th front) and assign domination rank $\mathrm{R}_{\mathrm{p}}=\mathrm{i}$.

vii. For each $p \epsilon \mathrm{F}_{\mathrm{i}}$ assign crowding distance to $p$.

viii. Increment front index by 1 ; i.e. $\mathrm{i}=\mathrm{i}+1$.

ix. $\quad$ For each $p \in \mathrm{F}_{\mathrm{i}-1}$, visit each $q \in \mathrm{S}_{\mathrm{p}}$ and decrement $n_{q}$ by 1 , if by doing so, $n_{q}$ becomes 0 then add $q$ into the set $\mathrm{F}_{\mathrm{i}}$ ( $q$ belonging to front i, $R_{q} \quad=\mathrm{i}$ ).
} 
x. Repeat step viii to find subsequent fronts.

xi. Perform recombination as follows (steps "a" - "j" below).

(a) Set child population $Q_{t}=\varnothing$.

(b) Use binary tournament selection to select two parents from population $\mathrm{P}_{\mathrm{t}}$.

(c) With probability $\mathrm{P}_{c}$, perform uniform crossover on the selected parents to evolve with a child $\mathrm{p}$.

(d) With probability $\mathrm{P}_{\mathrm{m}}$, perform mutation by perturbing the gene of a selected component (component $\mathrm{i}$ ) with a value between

1 and $\alpha_{\text {imax }}$

(e) Add $p$ to $Q_{t}$; i.e. $Q_{t}=Q_{t} \cup p$.

(f) If the size of $\mathrm{Q}_{t}$ is not equal to $\mathrm{N}$, then go to step " $\mathrm{b}$ ".

(g) $\forall p \in Q_{t}$, configure the variant of the system model with $p$. The values of objective functions (unavailability and cost) are also calculated.

(h) $\quad \mathrm{P}_{\mathrm{t}}$ and $\mathrm{Q}_{t}$ are combined into $\mathrm{B}_{\mathrm{t}}$; i.e. $\mathrm{B}_{\mathrm{t}}=\mathrm{P}_{\mathrm{t}} \cup \mathrm{Q}_{\mathrm{t}}$ and $\mathrm{B}_{\mathrm{t}}$ is sorted based on non-domination.

(i) From $2 \mathrm{~N}$ solutions (combination of $\mathrm{P}_{\mathrm{t}}$ and $\mathrm{Q}_{\mathrm{t}}$ ) in $\mathrm{B}_{\mathrm{t}}, \mathrm{N}$ best solutions comparison [13] to form $\mathrm{P}_{\mathrm{t}+1}$.

(j) Increment population index by 1 ; i.e. $\mathrm{t}=\mathrm{t}+1$.

xii. If maximum generation is not reached then go to iv else terminate giving the set of PM individuals in the first front $\mathrm{F}_{1}$ as the solution.

\section{Case Study}

The formulated optimisation is demonstrated on a model of an aircraft wheel brake system (AWBS) shown in Figure 1. HiP-HOPS, a well established dependability analysis tool which was first developed by Papadopoulos and McDemid[14] was used. Each component of the AWBS was annotated with failure expressions in HiP-HOPS. The expressions were simple and are as observable at the output ports of the components. Hence, the analysis is focused on omission of function.

The AWBS model is adapted from Sharvia[12]. The AWBS provides safe baking for the aircraft during taxiing. Safe braking means that there is supply of correct pressure to the brake actuator or the wheel brake system (WBS) seen in Figure 1. This way, skidding or taxiing beyond or before expected point could be prevented. The wheel brake system also prevents the occurrence of unintended aircraft motion, especially when parked.

The AWBS consists of two primary hydraulic pumps; Green and Blue. In normal mode of braking, the Green pump provides the required hydraulic pressure while the Blue pump provides pressure in alternative mode. The alternative mode becomes active when failure occurs in normal mode. The Green valve and Blue valve control pressure from the Green pump and Blue pump respectively. In normal mode the Green valve and Blue valve are both opened to provide constant stream of pressure to the Selector valve. However, only one of the two redundant hydraulic lines is selected by the Selector valve to prevent a scenario where both provide braking pressure.

In a normal braking mode, the brake system control unit (BSCU) can receive as input the Brake pedal position which it processes to produce control signals for braking. The BSCU also receives several other input signals which are continuously being monitored. These inputs indicate certain critical aircraft and system (AWBS) states so that the correct braking function is achieved to improve fault tolerance mechanism. The BSCU basically computes braking and anti-skid commands and transmits the signal to the appropriate 
braking channel. The Aircraft speed and Deceleration rate are used when Auto brake is true.

The Selector valve receives braking pressure from the Green pump and in addition to the control signal received from the BSCU, brake pressure is further transmitted to the CMD/AS meter valveG which measures the amount of brake pressure and adjusts the valve position to output the required amount of pressure based on the command issued by the BSCU. The brake pressure is further transmitted to the WBS through normal pressure NormalP.

Should failure exist in control pressure which emanates from CMD/AS meter valveG, a signal is sent to the BSCU to put the AWBS into alternative mode and the braking process continues in this mode. In addition, the AWBS enters alternative mode when (i) Green pump produces pressure below threshold or pressure is omitted, or (ii) when any other failure occur along the Green pump line. Once an alternative mode is activated, an OnAlternative signal is sent to inform the Selector valve to ignore any pressure from the Green valve. Once the AWBS goes into alternative mode, reverting to normal mode is impossible during the mission time of the aircraft.

The Selector valve in alternative mode receives braking control pressure from the Blue pump in addition to brake control signal from the BSCU. Brake pressure is then transmitted to the CMD/AS meter valveB which also measures the amount of brake pressure and adjusts the valve position to output the required amount of pressure based on the command issued by the BSCU. The brake pressure is further transmitted to the WBS through alternative pressure AlternativeP.

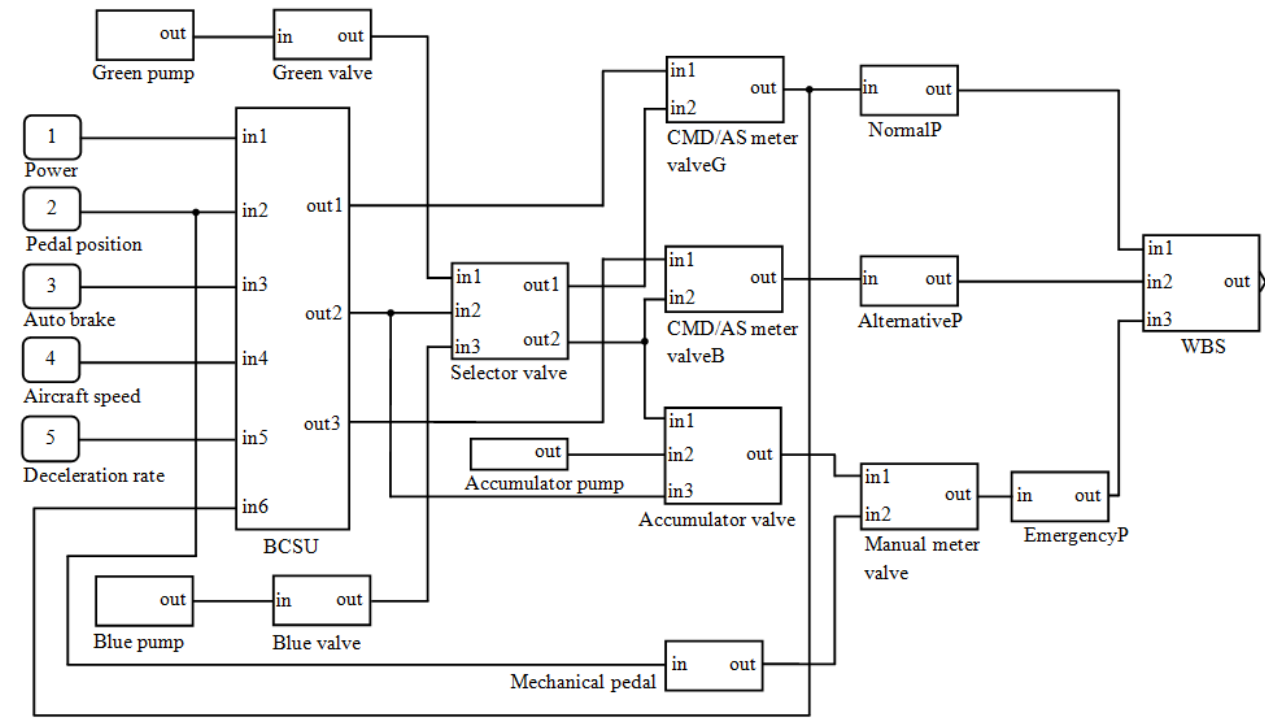

Figure 1. Aircraft Wheel Brake System

As an increased safety measure, the AWBS comprise of an Accumulator valve which continuously receives pressure from Accumulator pump. The Accumulator valve also receives control signal from the BSCU in order to be informed of what mode is in force. In Alternative mode primary channel of output is given to the CMD/AS meter valveB and the Accumulator valve is left redundant. However when there is no pressure from the Selector valve or the pressure falls under threshold when the AWBS is in Alternative mode, the AWBS enters emergency mode and pressure from the Accumulator pump is released to the Accumulator valve and the braking pressure is transmitted to the Manual meter valve. The Manual meter valve also receives as input, pressure from Mechanical pedal which serves as an extra safety measure. The Manual meter valve regulates pressure from the Selector valve and the Accumulator pump. The pressure is further transmitted to the WBS through emergency pressure EmergencyP. 


\section{Evaluations}

Components failure is assumed to follow Weibull distribution characteristics. Therefore, the defined optimisation problem was evaluated using the following parameters.

Weibull scale $=25000$ (assumed to be the useful life/risk time of the system)

Weibull shape parameter $=2$ (failure rate is assumed to increase with time)

Weibull location parameter $=0$ (components are assumed to be new when system was new)

In addition to the Weibull specific parameters, the optimisation was run through 400 generations and the following components were subjected to IPM policy while the rest to PPM policy: Green Pump, Blue Pump, Green Valve, Blue Valve, Accumulator Pump, Accumulator Valve, Selector Valve.

The results are discussed next.

\subsection{Results}

The optimisation run through 400 generations produced 4046 optimal PM schedules. Among these optimal PM schedules, a system engineer could select which one to implement based on requirements; such as within a cost and unavailability bracket or a schedule that is closest to a specified value for cost and unavailability. Figure 2 shows the Pareto frontier of the solutions. At the 400thgeneration, optimal PM schedules were also found which implies that it is likely that beyond the 400th generation more optimal PM schedules will be found.

The optimal PM schedules at the further end of each axis in the Pareto frontier is here defined as the terminal optimal schedules, and the values of both are thus discussed. The optimal PM schedule found with the lowest cost value of 51277.4 and highest unavailability value of 0.151447 was in generation 7. Similarly, the optimal PM schedule found with the highest cost value of 82309.7 and lowest unavailability value of 0.104925 was found in generation 143. This implies that the objective space in terms of cost is 31032.3 (82309.7 - 51277.4) and $0.046522(0.151447$ - 0.104925) in terms of unavailability.

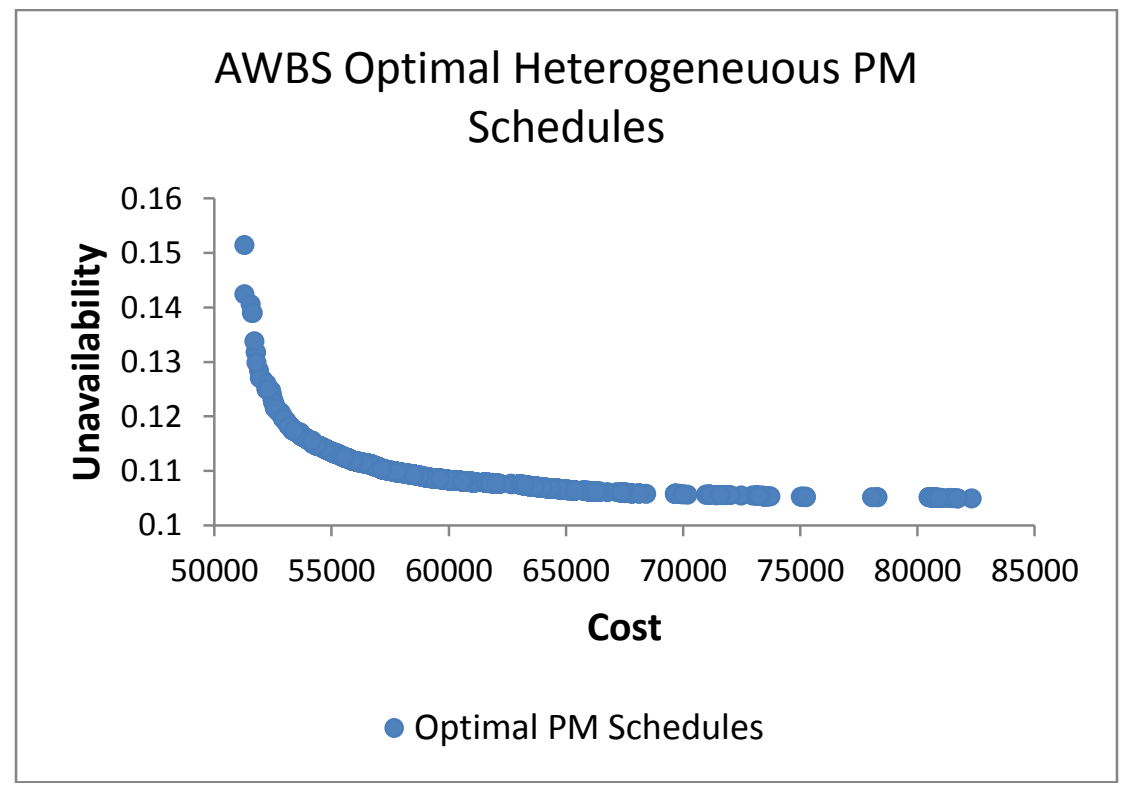

Figure 2. Optimal Heterogeneous PM Schedules 
Figure 2 shows the possible configurations of the system with respect to heterogeneous PM policy. As mentioned earlier, it is easier to assume PPM on all components of the system and to carry out the evaluations and thus, the optimisation. Therefore, it will be useful to also perform PPM optimisation on the AWBS under the same specifications as the heterogeneous PM policy and to discuss the two results. The results of the PPM optimisationare shown in Figure 3.

PPM optimisation was allowed to run up to the 400th generation and produced 1128 PPM schedules. The Pareto frontier of the PPM schedules is as shown in Figure 3. The optimal PPM schedule found with the lowest cost value of 48590 and highest unavailability value of 0.304612 was in generation 22. Similarly, the PPM schedule with the highest cost value of 137640 and lowest unavailability value of 0.0104905 was found in generation 369. The objective space in terms of cost is 89050 (137640 - 48590) and $0.2941215(0.304612-0.0104905)$ for unavailability. Table 1 presents the objective functions value of the terminal optimal schedules of both Figure 2 (heterogeneous PM schedules) and Figure 3 (PPM schedules) while Table 2 presents the differences in object space between heterogeneous PM and PPM policy.

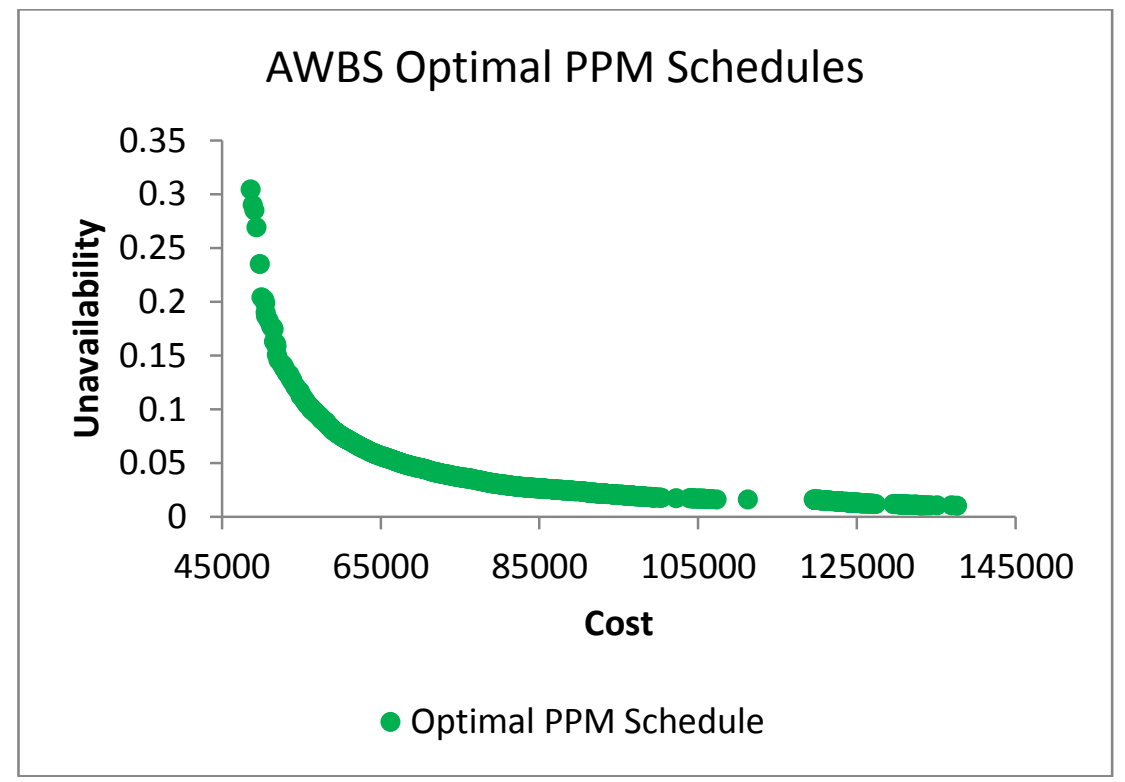

Figure 3. Optimal PPM Schedules

Table 1. Objective Functions Value of Terminal Optimal Schedules of Figures 2 and 3

\begin{tabular}{|l|l|l|}
\hline & Heterogeneous PM (HPM) & PPM \\
\hline Highest Cost & 82309.7 & 137640 \\
\hline Lowest Unavailability & 0.104925 & 0.0104905 \\
\hline Lowest Cost & 51277.4 & 48590 \\
\hline Highest Unavailability & 0.151447 & 0.304612 \\
\hline
\end{tabular}

As expected, Table 1 shows that PPM scheduling optimisation produced the PM schedule with the highest costand the lowest unavailability value. 
Table 2. Difference in Objective Space between Heterogeneous PM and PPM Schedules

\begin{tabular}{|c|l|l|}
\hline & Cost & Unavailability \\
\hline PPM & 89050 & 0.2941215 \\
\hline $\begin{array}{l}\text { Heterogeneous PM } \\
\text { (HPM) }\end{array}$ & 31032.3 & 0.046522 \\
\hline Difference (PPM - HPM) & 58017.7 & 0.2476 \\
\hline
\end{tabular}

Although the PPM optimisation has greater objective space, it produced less number of optimal PM schedules. This implies that the heterogeneous PM optimisationas illustrated in this paper and on the specific case study, produced schedules with lower crowding distance between successive optimal schedules in the Pareto frontier.

\section{Conclusions}

Real maintenance problem of engineering systems consists of components whose maintenance actions will result into renewal while others into rejuvenation to a certain degree of improvement. The former refers to perfect preventive maintenance (PPM) while the latter imperfect preventive maintenance (IPM). A maintenance policy which combines the two is referred to as heterogeneous preventive maintenance. This paper has defined the optimisation of heterogeneous preventive maintenance (PM) policy and demonstrated its applicability via a case study. HiP-HOPS was used as the analysis tool. The optimal schedules show trade-offs between cost and unavailability where an engineer can select a schedule based on requirements. Notability, this paper showed that the key to evaluating a heterogeneous PM policy is in defining the evaluation models for perfect preventive maintenance (PPM) and imperfect preventive maintenance (IPM) respectively. A further comparison between optimal heterogeneous PM schedules and optimal PPM schedules showed that PPM optimal schedules has wider objective space although more solutions were found under heterogeneous PM optimisation, implying lower crowding distance between successive optimal schedules in the Pareto frontier.

Further work will be required to automatically generate optimal schedules within a set bracket value for the objective functions value (cost and unavailability).

\section{References}

[1] J. Zhao, A. H. C. Chan, C. Roberts and K. B. Madelin, "Reliability Evaluation and Optimisation of Imperfect Inspections for a Component with Multi-defects", Reliability Engineering and System Safety, vol. 92, (2007), pp. $65-73$.

[2] S. H. Nggada, D. J. Parker and Y. I. Papadopoulos, "Dynamic Effect of Perfect Preventive Maintenance on System Reliability and Cost Using HiP-HOPS", IFAC-MCPL, 5th Conference on Management and Control of Production and Logistics, IFAC Proceedings Volumes (IFAC-PapersOnline), (2010); Coimbra, Portugal.

[3] S. H. Nggada, Y. I. Papadopoulos and D. J. Parker, "Extending HiP-HOPS with Capabilities of Planning Preventative Maintenance, In: Strategic Advantage of Computing Information Systems in Enterprise Management", Volume Containing Revised Selected Papers from the International Conference in Computer Systems and Information Systems, (2010).

[4] A. Sanchez, S. Carlos, S. Martorell and J. F. Villanueva, "Addressing Imperfect Maintenance Modelling Uncertainty in Unavailability and Cost Based Optimization", Reliability Engineering and System Safety, vol. 94, no. 1, (2009), pp. 23-32.

[5] S. Martorell, A. Sanchez and V. Serradell, "Age-Dependent Reliability Model Considering Effects of Maintenance and Working Conditions”, Reliability Engineering and System Safety, vol. 64, no. 1, (1999), pp. 19-31.

[6] Y. Tsai, K. Wang and H. Teng, "Optimizing Preventive Maintenance for Mechanical Components using Genetic Algorithms”, Reliability Engineering and System Safety, vol. 74, no. 1, (2001), pp. 89-97. 
[7] S. Goto, Y. Onizuka and M. Nakamura, "Optimal Maintenance Scheduling by Average System Availability Through Appropriate Segmentation of Maintenance Intervals", International Journal of Innovative Computing, Information and Control, vol. 2, no. 5, (2006), pp. 1083-1096.

[8] Y. Tsai, K. Wang and L. Tsai, "A Study of Availability-centered Preventive Maintenance for Multicomponent Systems", Reliability Engineering and System Safety, vol. 84, (2004), pp. 261-270.

[9] S. Sheu, Y. Lin and G. Liao, "Optimum Policies for a System with General Imperfect Maintenance", Reliability Engineering and System Safety, vol. 91, no. 3, (2006), pp. 362-369.

[10] A. Birolini, "Reliability Engineering: Theory and Practice (5th Ed)", Springer, Berlin, (2007).

[11] T. Jin and D. W. Coit, "Approximating Network Reliability Estimates Using Linear and Quadratic Unreliability of Minimal Cuts", Reliability Engineering and System Safety, vol. 82, no. 1, (2003), pp. 41-48.

[12] S. Sharvia, "Integrated Application of Compositional and Behavioural Safety Analysis", PhD Thesis, University of Hull, (2010).

[13] S. Favuzza, M. G. Ippolito and E. R. Sanseverino, "Crowded Comparison Operators for Constraints Handling in NSGA-II for Optimal Design of the Compensation System in Electrical Distribution Networks", Advanced Engineering Informatics, vol. 20, no. 2, (2006), pp. 201-211.

[14] Y. I. Papadopoulos and J. A. McDermid, "Hierarchically Performed Hazard Origin and Propagation Studies", 18th International Conference in Computer Safety, Reliability and Security, (1999); Toulouse, France.

[15] S. H. Nggada, "Determining System Design Space Relative to its Architecture and Preventive Maintenance Policy”, International Journal of Control and Automation, vol. 7, no. 4, (2014), pp. 165174.

\section{Author}

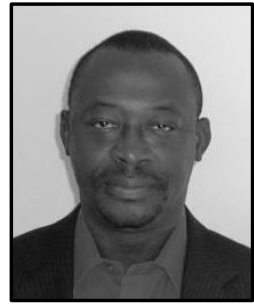

Shawulu Hunira Nggada, He has PhD in Computer Science and PGDp in Research Training both from University of Hull - UK, MSc Software Engineering from University of Bradford - UK and B. Tech (Hons) in Computer Science from Abubakar Tafawa Balewa University, Nigeria. He has Chartered IT Professional membership of BCS The Chartered Institute for IT, Chartered Engineer membership of Engineering Council - UK. He is also a member of Nigeria Computer Society, IEEE Reliability Society and ACM. He is currently a Deputy Director in Computer Science at Namibia University of Science and Technology. 